\title{
Should Age be Considered When Proposing Decompressive Hemicraniectomy in Malignant Stroke, and If so Where is the Limit?
}

\author{
Dimitre Staykov $\cdot$ Stefan Schwab
}

Published online: 6 June 2012

(C) Springer Science+Business Media, LLC 2012

Malignant middle cerebral infarction is defined as a clinical deterioration from hemispheric swelling and is named "malignant" because it is rapidly injurious to the patient. Recommendations for early decompressive hemicraniectomy (DHC) in malignant middle cerebral artery infarction (mMCAI) in the clinical routine are based on data from three European randomised controlled trials (DECIMAL, DEcompressive Craniectomy In MALignant middle cerebral artery infarcts [1]; DESTINY, DEcompressive Surgery for the Treatment of malignant Infarction of the middle cerebral artery [2]; and HAMLET, Hemicraniectomy After Middle cerebral artery infarction with Life-threatening Edema Trial [3]) and two pooled meta-analyses [3, 4]. The results clearly demonstrated that DHC is more than just an optional intervention in mMCAI as mortality was consistently significantly lower in patients who underwent surgery across all three trials, as compared with controls. None of the trials or meta-analyses were able to show significant improvement in functional outcome with DHC when the predefined dichotomization between a modified Rankin scale score (mRS) of 0-3 and 4-6 was used [3, 5]. However, a post hoc meta-analysis of the pooled data including totally 109 patients indicates that early surgery $(<48 \mathrm{~h}$ after symptom onset) may also result in a higher proportion of patients with a mRS of 4 or lower, as compared to best medical treatment [3]. One of the unresolved and important questions after DECIMAL, DESTINY, and HAMLET is, if there is an age limit from which on the benefits of DHC are nullified.

The difficulty to derive any conclusions on this age issue is because the clinical trials included only patients younger

D. Staykov $(\bowtie) \cdot$ S. Schwab

Department of Neurology, University Hospital Erlangen,

Schwabachanlage 6, 91054 Erlangen, Germany

e-mail: dimitre.staykov@uk-erlangen.de than 60 years; however, a major proportion of the patients suffering mMCAI belong to an older age cohort [6]. Observational studies reporting on older patients treated with DHC are rather skeptical showing improved survival achieved at the cost of poor functional outcome and dependency [6-8].

In the current issue of Neurocritical Care, Zhao and colleagues report the results of a Chinese randomised controlled study [9] which investigated the effect of early decompressive hemicraniectomy after large mMCAI in patients aged 18-80 years. Forty-seven patients were included in the trial and 24 of them were randomized to early DHC. In line with the findings of the 3 European RCTs, surgical treatment resulted in significantly lower mortality at six months (12.5 vs. $60.9 \%)$ and one year after symptom onset (16.7 vs. $69.6 \%$ ). In addition, Zhao et al. could demonstrate a significant benefit from surgery as emphasized by the higher rate of patients with a mRS of 5 or 6 six months (33.3 vs. $82.6 \%$ ) and one year after symptom onset ( 25 vs. $87 \%$ ). The most interesting aspect of this study is the age distribution of the included participants. Of totally 47 patients enrolled, 29 were older than 60 years and 16 of them were randomized to surgical treatment. Subgroup analyses including only patients older than 60 years yielded similar results. A significant benefit of surgery was found in this older cohort considering mortality (6 months: 12.5 vs. $61.5 \%$; 1 year: 18.8 vs. $69.2 \%$ ), as well as poor outcome, defined as mRS > 4 (6 months: 31.2 vs. $92.3 \%$; 1 year: 37.5 vs. $100 \%)$. Based on those findings, the authors come to the optimistic conclusion that DHC is beneficial for patients aged 61-80 years although there seems to be an increased likelihood for those patients to survive with moderately severe disability (mRS 4).

As Zhao et al. state in the discussion of their article, those results are "exciting," providing the first outcome 
data from a randomized controlled trial for DHC in older patients with mMCAI. But, is this the answer to the question if there is an upper age limit for surgery after mMCAI? Do those results justify a recommendation for decompressive surgery in an 80-year old? We do not share the authors' optimism. In light of the hitherto existing evidence from large observational studies, uniformly showing that age does play a role as a strong negative prognostic factor in malignant stroke treated with DHC [10], in neurocritical care [11, 12], and in critical care in general $[13,14]$, we think that a premature conclusion based on data from a handful of patients is not warranted. Furthermore, approximately two-thirds (18/ 29 ) of the patients aged $>60$ in the study of Zhao et al. were 61-70 years old. Only 11 patients (4 randomized to surgery) belonged to the older subcohort aged 71-80 years. Looking at those relatively small patient numbers, it is rather surprising that the authors could see such highly significant results. At this point, it appears more appropriate to cautiously state that the study may indicate that an age limit for surgery after mMCAI may lie above 60 years. The fact that the present study was registered retrospectively after its completion (http://www.chictr.org/en/), a rather unusual approach for a current phase III trial meeting international standards, should also be considered when those results are interpreted. Moreover, after the meta-analysis of the RCTs, DECIMAL, DESTINY, and HAMLET were published in March 2007 [4, 7] early DHC for mMCAI in patients younger than 60 years has been widely accepted as a lifesaving treatment based on robust class I evidence. Therefore, the inclusion and randomization of patients younger than 60 years by Zhao et al. starting at a later time point raises questions on the ethical standards applied to this study.

Hopefully, the issue of the age limit in DHC can be resolved when DESTINY 2 is published [15]. This European multicenter RCT has enrolled over 100 patients with mMCAI who were older than 60 years.

Clearly, we need more hard data before we are pursuing DHC in elderly patients who might be poor candidates for aggressive rehabilitation. In day to day practice, many family members may feel that such a major neurosurgical procedure is not warranted and we need more convincing data to go that path.

\section{References}

1. Vahedi K, Vicaut E, Mateo J, et al. Sequential-design, multicenter, randomized, controlled trial of early decompressive craniectomy in malignant middle cerebral artery infarction (DECIMAL Trial). Stroke. 2007;38:2506-17.

2. Juttler E, Schwab S, Schmiedek P, et al. Decompressive surgery for the treatment of malignant infarction of the middle cerebral artery (DESTINY): a randomized, controlled trial. Stroke. 2007; 38:2518-25.

3. Hofmeijer J, Kappelle LJ, Algra A, Amelink GJ, van Gijn J, van der Worp HB. Surgical decompression for space-occupying cerebral infarction (the hemicraniectomy after middle cerebral artery infarction with life-threatening edema trial [HAMLET]): a multicentre, open, randomised trial. Lancet Neurol. 2009;8: 326-33.

4. Vahedi K, Hofmeijer J, Juettler E, et al. Early decompressive surgery in malignant infarction of the middle cerebral artery: a pooled analysis of three randomised controlled trials. Lancet Neurol. 2007;6:215-22.

5. Mitchell P, Gregson BA, Crossman J, et al. Reassessment of the HAMLET study. Lancet Neurol. 2009;8:602-3. (author reply 3-4).

6. Holtkamp M, Buchheim K, Unterberg A, et al. Hemicraniectomy in elderly patients with space occupying media infarction: improved survival but poor functional outcome. J Neurol Neurosurg Psychiatry. 2001;70:226-8.

7. Uhl E, Kreth FW, Elias B, et al. Outcome and prognostic factors of hemicraniectomy for space occupying cerebral infarction. J Neurol Neurosurg Psychiatry. 2004;75:270-4.

8. Rabinstein AA, Mueller-Kronast N, Maramattom BV, et al. Factors predicting prognosis after decompressive hemicraniectomy for hemispheric infarction. Neurology. 2006;67:891-3.

9. Zhao J, Su YY, Zhang Y, et al. Decompressive Hemicraniectomy in Malignant Middle Cerebral Artery Infarct: A Randomized Controlled Trial Enrolling Patients up to 80 Years Old. Neurocrit Care 2012

10. Gupta R, Connolly ES, Mayer S, Elkind MS. Hemicraniectomy for massive middle cerebral artery territory infarction: a systematic review. Stroke. 2004;35:539-43.

11. Kiphuth IC, Schellinger PD, Kohrmann M, et al. Predictors for good functional outcome after neurocritical care. Crit Care. 2010;14:R136.

12. Broessner G, Helbok R, Lackner P, et al. Survival and long-term functional outcome in 1,155 consecutive neurocritical care patients. Crit Care Med. 2007;35:2025-30.

13. Vasilevskis EE, Kuzniewicz MW, Cason BA, et al. Predictors of early postdischarge mortality in critically ill patients: a retrospective cohort study from the California intensive care outcomes project. J Crit Care. 2011;26:65-75.

14. Goldhill DR, Sumner A. Outcome of intensive care patients in a group of British intensive care units. Crit Care Med. 1998; 26:1337-45.

15. Decompressive surgery for the treatment of malignant infarction of the middle cerebral artery 2, ISRCTN21702227 (Accessed 9 Sept 2010, at http://www.controlled-trials.com/ISRCTN217022 27/DESTINY). 